\title{
La teoría del diálogo platónico: antecedentes en y a partir de la hermenéutica schleiermacheriana
}

Carolina Delgado*

\begin{abstract}
Resumen: En este artículo, ofrezco una breve exposición de la teoría del diálogo platónico tal como ésta aparece en el paradigma schleiermacheriano. En concreto, estudio la interpretación de la forma del diálogo desde la perspectiva hermenéutica que el filósofo alemán elabora en sus Lecciones sobre hermenéutica y en Los discursos sobre hermenéutica; a modo de anexo, ofrezco también una relación sucinta de los principales estudios actuales relativos al tema.
\end{abstract}

Palabras clave: Diálogo, hermenéutica, Platón, Schleiermacher.

\begin{abstract}
This paper offers offer a brief account of the Theory of Platonic Dialogue as it appears in Schleiermacher's paradigm. Specifically, the author studies the interpretation of the form of dialogue from a hermeneutic perspective, which the German philosopher develops in his Lessons on Hermeneutics and Lectures on Hermeneutics. The attachment includes a concise list of the most important current studies on the subject.
\end{abstract}

Key words: Dialogue, Hermeneutics, Plato, Schleiermacher

Résumé: Je propose, dans cet article, une brève présentation de la théorie du dialogue platonicien telle que celle-ci apparaît dans le paradigme schleiermachérien. Concrètement, j'étudie l'interprétation de la forme du dialogue depuis la perspective herméneutique que le philosophe allemand élabore dans ses Leçons d'herméneutique et Les Discours sur l'herméneutique; en guise d'annexe, je propose également une liste succincte des principales études actuelles concernant ce sujet.

Mots-clés: Dialogue, herméneutique, Platon, Schleiermacher.

* Doctora en Filosofía. Becaria del DAAD en la Universidad de Bonn, Alemania.

Recibido: 2009 - 09 - 03 (acdelgado04@hotmail.com).

Aprobado: 2009 - 12 - 09 
La obra platónica es, al mismo tiempo que eminentemente filosófica, una obra literaria. Como ejemplo de ese carácter literario se puede señalar la forma del diálogo con la que Platón comunica su pensamiento, la cual ha sido con frecuencia objeto de la investigación en el ámbito de los estudios platónicos más actuales ${ }^{1}$. El punto de referencia obligado para reseñar las líneas interpretativas más actuales resulta, sin embargo, el así denominado 'paradigma' interpretativo de F. Schleiermacher ${ }^{2}$. En las siguientes páginas, presento - a modo de panorámica general - una exposición de la teoría del diálogo platónico tal como emerge de la perspectiva hermenéutica del filósofo alemán; a modo de anexo, ofrezco una relación sucinta de los principales estudios actuales relativos al tema.

\section{Contextualización: Schleiermacher y la obra platónica}

Fue F. Schleiermacher quien asumió de manera prominente la propuesta que hiciera ya Leibniz (Scientia Generalis Characteristica, 1704) de regresar a Platón en una lectura directa de

1 Por ejemplo, la investigación se ha centrado en el contexto literario de los diálogos platónicos: D. Clay, "The Origins of the Socratic Dialo-

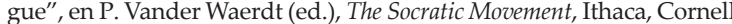
University Press, 1994 y Ch. Kahn, Plato and the Socratic Dialogue. The Philosophical Use of a Literary Form, Cambridge, Cambridge University Press, 1996; en su estructura: H. Thesleff, Studies in Platonic Chronology, Helsinki, Societas Scientarum Fennica, 1982; en su función: K. Gaiser, Platone come scrittore filosofico. Saggi sull'ermeneutica dei dialoghi platonici, Napoli, Istituto italiano per gli studi filosofici, 1984; en la importancia de la forma del diálogo como parte importante de la concepción platónica de filosofía: Ch. Rowe, Plato, Brighton, Harvester, 2003; F. J. González, The Third Way. New Directions in Platonic Studies, London, Lanham, 1995; C. Gill y M. McGabe (eds.), Form and Argument in Late Plato, Oxford, Clarendon Press, 1996; A. W. Nightigale, Genres in Dialogue. Plato and the Construct of Philosophy, Cambridge, Cambridge University Press, 1995; Ch. Griswold, (ed.), Platonic Writings. Platonic Readings, New York, Routledge, 1988.

2 Así H. J. Krämer, Platone e i fondamenti della metafisica. Saggio sulla teoria dei principi e sulle dottrine non scritte di Platone, Milano, Vita e Pensiero, 1993, 4 ed.; y G. Reale, Per una nuova interpretazione di Platone. Rilettura della metafisica der grandi dialoghi alla luce delle 'Dottrine non scritte', Milano, Vita e Pensiero, 1991 (11 ${ }^{\underline{a}}$ ed.). sus diálogos ${ }^{3}$. Como es sabido, Schleiermacher inició con F. Schlegel el proyecto común-que, sin embargo, luego continuaría solo- de traducir al alemán la obra completa de Platón ${ }^{4}$. Esta traducción comprende el entero Corpus Platonicum, es decir, los veinticuatro diálogos hasta hoy tenidos por auténticos (con excepción de Timeo, Critias, Leyes y Cartas) y los siete inauténticos, pero transmitidos bajo la autoría de Platón ${ }^{5}$. Además de su traducción, Schleiermacher elaboró introducciones a cada uno de los diálogos, que -junto con las lecciones de historia de la filosofía dictadas entre 1809 y 1823- constituyen el material fundamental para reconstruir su interpretación de la filosofía platónica ${ }^{6}$.

Como ha sido indicado, en la interpretación de Schleiermacher sobre Platón pueden distinguirse cuatro fases ${ }^{7}$. La primera etapa se extiende desde su relación con Schlegel hasta que asume personalmente el trabajo de traducción (aprox. 1798-1803); la segunda, desde esta primera fase hasta que concluye la traducción de la obra platónica (aprox. 1803-1809); la terce-

3 Así M. Erler, Die Philosophie der Antike. Band 2/2. Platon, hrsg. H. Flashar, Überweg, Basel, Schwabe, 2007.

4 Sobre el proyecto común de Schlegel y Schleiermacher, véase J. Lamm, "Schleiermacher as Plato Scholar", The journal of religion, 80, II, 2000, 206-239; A. Arndt, „Schleiermacher und Platon“, en F.D.E. Schleiermacher, Über die Philosophie Platos. Geschichte der Philosophie. Vorlesungen, über Sokrates und Platon (zwischen 1819 und 1823). Die Einleitungen zur Übersetzung des Platon (1804-1828), hrsg. P. Steiner mit Beiträgen von A. Arndt und J. Jantzen, Hamburg, Felix Meiner Verlag, 1996.

$5 \mathrm{Al}$ respecto, véase P. Steiner, „Zur Kontroverse um Schleiermachers Platon", en F.D.E. Schleiermacher, Über die Philosophie Platos. Geschichte der Philosophie. Vorlesungen, über Sokrates und Platon (zwischen 1819 und 1823). Die Einleitungen zur Übersetzung des Platon (1804-1828), hrsg. P. Steiner mit Beiträgen von A. Arndt und J. Jantzen, Hamburg, Felix Meiner Verlag, 1996.

6 Tanto la introducción general a la traducción de la obra platónica y las introducciones a los diálogos singulares como las lecciones de historia de la filosofía están recogidas en: F.D.E. Schleiermacher, Über die Philosophie Platos. Geschichte der Philosophie. Vorlesungen über Sokrates und Platon (zwischen 1819 und 1823). Die Einleitungen zur Übersetzung des Platon (1804-1828), hrsg. P. Steiner mit Beiträgen von A. Arndt und J. Jantzen, Hamburg, Felix Meiner Verlag, 1996. Al incluir referencias a la Introducción general (Einleitung I 1), citaré la numeración de la primera edición (A)

7 Steiner, „Zur Kontroverse...", ob. cit., p. 26. 
ra abarca la época de las lecciones sobre Platón y Sócrates (aprox. 1809-1820); y, finalmente, la cuarta etapa abarca la revisión y modificación de la traducciones de Platón y de sus correspondientes introducciones (aprox. 1817-1828).

Con su trabajo de traducción y con sus introducciones a los diálogos, Schleiermacher da un impulso totalmente nuevo a la investigación en el ámbito de los estudios platónicos, que se funda en y, al mismo tiempo, alimenta la creación de una hermenéutica del diálogo ${ }^{9}$. En efecto, constituye indudablemente un mérito de Schleiermacher el haber llamado la atención sobre el hecho de que la forma del diálogo no es en la obra de Platón un mero ropaje externo, sino un elemento esencial en la comunicación de su filosofía ${ }^{10}$. Este marco teórico que emerge de la interpretación de la obra platónica y simultáneamente la sustenta, se encuentra formulada en los textos con sus Lecciones sobre hermenéutica ${ }^{11}$ y sintetizado en Los discursos sobre hermenéutica, compuestos por dos conferencias que Schleiermacher pronunció en 1829 en la Academia de las Ciencias de Berlín ${ }^{12}$. Voy a seguir principalmente estos discursos, aunque las referencias expresas a Platón son aquí marginales. En esta concepción de la tarea interpretativa encuentra su explicación el hecho de que Schleiermacher destacara la relevancia de la forma de diálogo en la que es transmitida la filosofía platónica ${ }^{13}$.

8 Me refiero a la revisión de la interpretación neoplatónica dominante hasta primera mitad del siglo XIX. Al respecto, véase Lamm, "Schleiermacher as...", ob. cit., p. 219.

9 Sobre el particular, véase A. Neschke-Hentschke, "Platonisme et tournant herméneutique au début du XIXe siècle en Allemagne", en A. Laks, y A. Neschke (ed.), La naissance du paradigme herméneutique. Schleiermacher, Humboldt, Boeckh, Droysen, Paris, Presses Universitaires de Lille, 1990.

10 Como explica Neschke ("Platonisme et...", ob. cit., p. 139), la forma del diálogo platónico había sido considerada incluso por precedentes inmediatos a Schleiermacher -como Eberhard, Brucker y Tennemann- un verdadero obstáculo para la comprensión del pensamiento filosófico de Platón. Por eso, aunque en esta Einleitung Schleiermacher no mencione nombres expresamente, hay que tener en cuenta que alude sin embargo a tales lecturas y se posiciona ante ellas críticamente. También Laks (La naissance..., ob. cit., p. 156).

11 Schleiermacher, Hermeneutik und Kritik, Frankfurt am Main, Suhrkamp, 1990.

12 Tales conferencias se titulan "Sobre el concepto de hermenéutica en relación a las indicaciones de F. A. Wolf y al manual de Ast".

13 Esta relevancia que Schleiermacher concede a la forma del diálogo para la verdadera comprensión de la filosofía platónica, puede verse en la siguiente declaración, que subraya por contrapartida la unidad

\section{Los discursos de hermenéutica}

Como es sabido, la tarea hermenéutica vigente en el entorno de Schleiermacher se había visto vinculada a la necesidad de apropiarse del sentido auténtico de textos de la antigüedad clásica y de la Sagrada Escritura con el fin de tornarlos cercanos a los contemporáneos. La praxis de interpretación presentaba, en este sentido, dos carencias que él acusa y que propone superar: en primer lugar, la restricción del objeto hermenéutico al campo exclusivo de los textos escritos; y, en segundo lugar, la concepción de la hermenéutica o bien como la mera aplicación de reglas más o menos sistematizadas pero que carecen de una adecuada comprensión de la naturaleza del pensamiento, del lenguaje y de su modo de relación, o bien -en otro extremo- como el acceso puramente intuitivo al pensamiento de un escritor a partir de su obra.

Mis dos guías (Wolf, Ast) me limitan de varias maneras; uno por el hecho de que sólo habla de escritores que deben ser comprendidos como si no pudiera acontecer lo mismo también en la conversación y el discurso percibido inmediatamente; el otro porque restringe rápidamente lo extraño a lo compuesto en una lengua extraña, y después a las obras del espíritu concebidas así, lo cual es de nuevo un campo todavía más restringido que el de los escritores en general ${ }^{14}$.

Respecto de la primera falencia, Schleiermacher reclama para la hermenéutica una ampliación del horizonte propio, ya que los ejemplos tan cotidianos como la conversación con un amigo, la lectura de una carta o del periódico hacen patente la necesidad de extender el campo de desenvolvimiento de dicho arte, incluyendo en el mismo todo ámbito que tenga que ver con la comprensión de un discurso extraño en sentido general y no tan sólo en los casos de escritos procedentes de la antigüedad clásica.

indisociable de la forma y el contenido en el diálogo. Schleiermacher, Einleitung I, 1, A17: „So werden jene keinesweges die Philosophie des Platon kennen lernen; denn wenn irgendwo, so ist in ihr Form und Inhalt unzertrennlich".

14 F.D.E. Schleiemacher, Los discursos sobre hermenéutica, introducción, traducción y notas de L. Flamarique, Pamplona, Universidad de Navarra, Servicio de Publicaciones, 1999, p. 61. 
Donde quiera que haya algo extraño en la expresión del pensar a través del discurso para quien lo percibe, allí hay una tarea que no puede ser resuelta sino con ayuda de nuestra teoría $<$ hermenéutica $>^{15}$.

El discurso (Rede) en tanto que expresado -ya sea en lenguaje oral como en el escrito- y la extrañeza-radicada, por ejemplo, en un idioma no compartido, en el espacio histórico diverso, en la variabilidad de dotaciones de sentido o énfasis de un texto- constituyen el objeto directo de la praxis hermenéutica ${ }^{16}$.

Con respecto a la extrañeza, la tarea de interpretación supone, por una parte, la presencia de dos sujetos, a saber, el autor del discurso y el intérprete (lector, interlocutor, amigo) y, por otra, una relación previamente dada entre estos sujetos que comparten una instancia más básica de identidad por la que es posible precisamente establecer la relación:

Si todo fuera completamente extraño, la hermenéutica no sabría alcanzar su trabajo; y lo mismo en el caso contrario, a saber, si nada fuera extraño entre el que habla y el que percibe, tampoco necesitaría enlazar nada ${ }^{17}$.

Ahora bien, cuando lo extraño en el discurso procede de un 'otro', cuyo aspecto definitorio radica en su conciencia libre de espíritu finito, la captación del discurso no puede ser vista por parte del intérprete como la simple identificación de conexiones necesarias. En cada caso, la tarea hermenéutica consistirá en detectar las distintas cargas semánticas de los mismos

15 Ibídem, p. ,59.

16 Schleiermacher, Hermeneutik und Kritik, ob. cit., p. 78: „Das Denken ist ein inneres Sprechen. Daraus erhellt aber auch positiv, dass die Sprache das Fortschreiten des Einzelnen im Denken bedingt. Denn die Sprache ist nicht nur ein Complexus einzelner Vorstellungen, sondern auch ein System von der Verwandschaft der Vorstellungen (...). Ebenso ist jede Rede immer nur zu verstehen aus dem ganzen Leben, dem sie angehört, d. h. da jede Rede nur als Lebensmoment des Redenden in der Bedingtheit aller seiner Lebensmomente erkennbar ist, und dies nur aus der Gesamtheit seiner Umgebungen, wodurch seine Entwicklung und sein Fortbestehen bestimmt werden, so ist jeder Redende nur verstehbar durch seine Nationalität und sein Zeitalter".

17 Schleiermacher, Los discursos..., ob. cit., p. 59. Este último aspecto evita agudizar inadecuadamente la idea de lo extraño, que desembocaría en la imposibilidad del establecimiento de cualquier tipo de respectividad (aún si la que se quiere establecer es la de una absoluta contradicción entre los polos a relacionar). términos en diversos pasajes, los grados de énfasis que utilizar, etc., para descubrir lo individual de su modo de expresión.

Y, si seguimos adelante y pensamos cómo es oportuno demostrar en las partes más amplias de un todo la coherencia de pensamientos a menudo tan difícil y averiguar la oculta concesión a indicaciones en cierto modo perdidas, entonces aquí no se trata únicamente, como Wolf lo expone, de componer y ponderar minuciosamente los momentos históricos, sino de adivinar el modo individual de combinación de un autor, que, si hubiera sido diferente, en la misma situación histórica y en la misma forma de disertación habría dado otro resultado ${ }^{18}$.

En algunas ocasiones, elementos más externos (por ejemplo, la ubicación de una obra en su género literario, el estilo habitual de su autor, el marco histórico) permiten contextualizar y obtener una primera familiarización provisional con el autor y su obra, de manera que en cierto modo se adivina la orientación de la misma, otorgando un acercamiento previo para su comprensión. Arranca así el contacto con el autor como individualidad que, mediante el trato frecuente, abrirá al intérprete de manera progresiva elementos clarificadores que lo acerquen a la comprensión adecuada del pensamiento comunicado en la obra.

Se trata de un tipo completamente distinto de certeza, también -como Wolf lo elogia de la crítica- más adivinatoria que resulta de que el intérprete se sumerja lo más posible en la entera constitución del escritor ${ }^{19}$.

Que el autor del discurso sea un espíritu a la vez que finito también constitutivamente libre -tanto en la autoría del discurso como en el mismo sujeto intérprete- hace que la comprensión del mismo sea inagotable, la cual supone una continua tentativa de captar el discurso aún en la conciencia de la imposibilidad de asirlo exhaustivamente. Debido a esta situación definitoria del trabajo hermenéutico, se prevé dividir la actividad de acceso al sentido de lo

18 Ibídem, p. 67.

19 Ibídem, p. 67. 
dicho en dos momentos ${ }^{20}$, a modo de estudio complementario y cooperativo que contribuya a garantizar la recepción de los múltiples elementos latentes en el discurso con la máxima riqueza posible. La alternancia de estos dos momentos conduce de modo paulatino a una comprensión de la obra hasta lograr la transparencia del pensamiento del autor. El paciente trabajo del intérprete sobre el discurso redunda en una afinidad y consiguiente apropiación del pensamiento del otro, de manera tal que la extrañeza inicial se torna poco a poco en una suerte de posterior identificación.

Manteniendo lo anterior, hay que destacar, sin embargo, que la relación de extrañeza entre los interlocutores no puede ser de ningún modo anulada, puesto que el distanciamiento temporal, la diferencia de idiomas o la perspectiva diversa con que se abordan los temas constan siempre en cualquier caso; y, por ello, habrá de tenerse en cuenta que jamás puede disolverse del todo el malentendido. La combinación de los procedimientos comparativo y adivinatorio como modos de acceso al discurso extraño supone, en un nivel, el intento de evitar el malentendido en la expresión del pensamiento del otro, pero al mismo tiempo -y en un nivel más básico- el reconocimiento de un permanente resto que subyace inevitablemente en el discurso, explicando la imposibilidad de acudir a la tarea hermenéutica como un mero procedimiento de aplicación de reglas que permitan el abordaje válido a la expresión humana.

La combinación de los procedimientos comparativo y adivinatorio pone en movimiento un trabajo en absoluto lineal, sino que descri-

20 Ibídem, p. 81. Según Schleiermacher, la interpretación incluye en sí dos momentos diversos pero complementarios y, por tanto, no sustituibles el uno por el otro. Por un lado, habrá de llevarse a cabo una denominada 'parte gramatical' de la interpretación con vistas a recoger los aspectos que definen la lengua en su objetividad y comprende un estudio histórico de los elementos constitutivos de la misma. Por otro, la 'parte psicológica' analiza el discurso en referencia al individuo y su modo propio de producción de pensamientos. Para ambas partes (la gramatical y la psicológica) se supone, a su vez, un doble momento metodológico, que contribuirá, mediante un movimiento alternante, a realzar los aspectos en que el discurso pone de manifiesto la inefable individualidad del autor (posibilidades, límites e innovaciones que el individuo genera en el todo mayor que lo contextualiza) y su ubicación en un marco explicativo más general (el individuo en relación con su lengua, pueblo, historia, género, estilo, etc.). Véase también Schleiermacher, Hermeneutik und Kritik, ob. cit., pp. 80, 101, 116, 178. be un círculo ${ }^{21}$. En el trazado de este círculo se dan pasos de ida y vuelta, progreso y retroceso correctivo de los avances, lo cual implica la aceptación de una relación previa entre el todo y las partes a interpretar. El ingreso a dicha estructura no nace de un punto cero, sino que supone el presentimiento de la totalidad en la que adquieren sentido las partes, presentimiento inicial que ha de ser confrontado más tarde $\mathrm{y}$, según el caso, rectificado o confirmado.

El trabajo de comprender e interpretar es un todo constante que se desarrolla paulatinamente, en cuyo transcurso posterior nos apoyamos mutuamente más y más, en la medida en que cada uno proporciona al resto puntos de comparación y analogías, pero (un todo) que en cada punto recomienza siempre de la misma manera, presintiendo ${ }^{22}$.

Un principio hermenéutico hace presente -en el trazado del círculo y en la orientación sobre el modo de ingreso al mismo- su poder explicativo, cuando define que el todo se comprende a partir de lo individual $\mathrm{y}$, al mismo tiempo, lo individual puede ser comprendido únicamente a partir del todo. En el caso del discurso, las partes o lo individual se describen como lo más pequeño en relación a un todo mayor del cual depende. Cada elemento, por tanto, habrá de ser comprendido según el mencionado principio desde su inclusión en el todo mayor que le corresponda. La comprensión de una palabra no se adquiere a modo de átomo inconexo, sino como unidad mínima de una frase que la dota de un determinado significado entre los varios que puede poseer o que la sitúa en un matiz más o menos fuerte. Así sucede con una frase singular respecto a una estructura de frases y, de ésta con respecto a varias estructuras, y luego de éstas en relación a la obra entera. Finalmente, la mejor interpretación de una obra concreta se obtendrá mediante la perspectiva más amplia que otorga el conocimiento de la obra total de un autor ${ }^{23}$.

$21 \mathrm{Al}$ respecto, véase Schleiermacher, Hermeneutik und Kritik, ob. cit., pp. 95 y 97.

22 Schleiermacher, Los discursos..., ob. cit., p. 87.

23 Schleiermacher se refiere esta visión de conjunto en su interpretación de los diálogos platónicos empleando la metáfora de la obra literaria como un cuerpo vivo. Véase Schleiermacher, Einleitung I 1, Phaidros, 
Este modo de interpretación supone una constante remisión de los elementos menores del discurso a su completitud, movimiento en el cual la propia referencialidad criba los posibles malentendidos. Pero esta posterior rectificación de un malentendido previo, supone que en el primer acercamiento al discurso el intérprete ha esbozado ya una línea inicial de sentido que le permite acceder a la comprensión del mismo y que, por su carácter anticipatorio-proyectivo, puede denominarse presentimiento. La dificultad que presenta el círculo hermenéutico descrito no aparece una vez que éste ha sido puesto en movimiento, sino que radica en el momento del comienzo y en el modo de ingresar al discurso, o con otras palabras en detectar dónde tiene origen el presentimiento.

En las obras escritas, el acercamiento inicial que permite obtener una idea de la organización general y, por tanto, la proyección de un primer sentido provisorio viene facilitado por la técnica de incluir habitualmente en el texto un prólogo o un índice o al menos un título. En algunos textos escritos no se cuenta con ello, pero en cambio es posible saber previamente el género al que dicho discurso pertenece (poesía, teatro, historia) o acceder a alguna información sobre el autor y su estilo. En el discurso oral, la comprensión del todo se logra hacia el momento final, cuando ya se han recibido todas y cada una de las partes. En cualquier caso, es de destacar que toda comprensión primera es siempre provisional e insegura. Por ello se requiere volver desde el final al principio y recomenzar de nuevo completando la interpretación. En la oscilación del ir y volver se capta la relación todo-partes y la combinación de la interpretación gramatical con la psicológica configura con mayor probabilidad un discurso en su sentido auténtico.

\section{Platón y la forma del diálogo}

La tarea hermenéutica es concebida por Schleiermacher, entonces, como el acceso com-

\footnotetext{
71: „In beiden Fällen erschiene ja das schöne und offenbar mit grossem Fleiss gearbeitete Werk auf eine höchst verwerfliche Weise verunstaltet, dass es wie ein lebendiges Wesen gebildet sein und einen mit dem Geiste angemessenen Körper mit verhältnissmässigen Teilen haben müsse".
}

prensivo al discurso extraño, ya sea de expresión oral o escrita. En esta descripción, el modelo dialógico, que favorece la vía de acceso al saber, adquiere también para el trabajo interpretativo un valor peculiar, ya que en el diálogo está presente también lo extraño que puede proceder del otro (interlocutor). El paradigma hermenéutico advierte en la conversación un modo de comprensión que, en parte, prioriza y después traslada a la esfera hermenéutica detectando en su ejercicio grandes similitudes y mostrando factibles aportaciones que el modelo dialógico ofrecería para dicha tarea. Schleiermacher se expresa en este sentido:

Quiero aconsejar al intérprete de obras escritas que practique diligentemente la interpretación de la conversación más significativa. Pues la presencia inmediata del hablante, la expresión viva que anuncia la participación de todo su ser espiritual, el modo como se desarrollan aquí los pensamientos desde la vida común, todo esto incita más allá de la consideración aislada de un solo escrito ${ }^{24}$.

Schleiermacher sugiere trasladar el modelo dialógico de conversación oral al campo más extenso de todo el ejercicio hermenéutico. La formulación de dicho modelo en el ámbito de la interpretación significa, en síntesis, que así como dos sujetos se entienden en el marco de una conversación en torno a algún asunto, así también el intérprete ha de abordar la comprensión del sentido de una obra. Reconociendo la presencia de una actividad interpretativa en cualquier tipo de comunicación oral, se acentúa la presencia del modo de comprensión dialógico en todo otro tipo de discurso, incluso en el del texto escrito.

En la traslación del modelo dialógico a todo trabajo interpretativo, se observa la configuración de un diagrama que posibilita determinar el funcionamiento de tal praxis hermenéutica. Dicho diagrama permite identificar tres elementos: ante todo la participación de dos sujetos entre los que transcurre una conversación en la cual se trata sobre un asunto. Si fuera

24 Schleiermacher, Los discursos...,ob. cit., p. 65. 
posible fijar uno de los sujetos exclusivamente como intérprete y el otro como interpretado, podría afirmarse que el objeto de la comprensión por parte de quien interpreta incluye dos niveles: el primero consiste en la correcta apropiación del pensamiento expresado por el interlocutor (la lógica interna de su discurso), donde el intercambio de preguntas y respuestas tiende al esclarecimiento de la justeza de las palabras y del significado captado por el intérprete en relación con la expresión intentada por su interlocutor; en el segundo nivel, la comprensión atiende a la adecuación del discurso con relación al asunto de que se trata, donde entra la posibilidad de un acuerdo o desacuerdo entre los participantes en el diálogo. En este nivel, la figura de la mutua refutación por parte de ambos sujetos ejerce la función correctiva de las perspectivas sustentadas. La tarea hermenéutica se orientaría específicamente al primer nivel del diálogo, es decir, a la comprensión del sentido del discurso. Mientras que el segundo nivel se reserva para el momento que podría llamarse dialéctico o de pretendido acceso al mundo. Schleiermacher subraya así que el trabajo interpretativo delinea un movimiento que va de un sujeto al discurso expresado y de éste al sujeto que lo expresa. Sólo desde él la interpretación vuelve a poner su atención sobre el asunto y sobre la corrección de las afirmaciones referidas al mismo.

El interés de Schleiermacher por la filosofía platónica -lejos de reducirse a un abordaje meramente histórico- responde a una aproximación 'sistemática' ${ }^{25}$. Me refiero a que, en la filosofía platónica, Schleiermacher destaca el empleo de una forma peculiar de comunicación del pensamiento (el diálogo literario), que remite a la conversación oral, esto es, al ámbito en el cual-según el pensador berlinés- se revela la estructura fundamental de la comprensión

25 Así Neschke-Hentsche («Platonisme et... », ob. cit., p. 138) afirma sobre la recepción de Platón que hace Schleiermacher: «accueille toutes les impulsions de type esthétique qu'il découvre à travers Schlegel, mais en les intégrant à une approche qui appartient en propre à l'orientation systématique de l'histoire de la philosophie. Autrement dit, Schleiermacher combine une interprétation systématique de Platon avec une thèorie de la forme de ces systèm, c'est-à-dire la dialectique, et avec un théorie de la présentation de cette forme dans les dialogue ». $(\text { Verstehen })^{26}$. La conversación -como he indicado ya-favorece la corrección de los fenómenos de disrupción (malentendidos) mediante las preguntas y respuestas o mediante la refutación de manera viva y mucho más eficaz que la exposición del pensamiento en un escrito ${ }^{27}$.

Puesto que en los diálogos literarios, Platón imita conversaciones orales, la forma de exposición de su obra no se reduce a un mero ropaje externo, sino que constituye el elemento esencial de la comunicación de su filosofía ${ }^{28}$.

Cuando se ha considerado más arriba el exterior de esta forma platónica y su esquema casi imprescindible como el ropaje dialógico, se lo ha supuesto como una característica especial que configura al diálogo platónico, pero sólo en cuanto ella (la forma dialógica) tiene el propósito de imitar mediante una interpretación viva las clases orales, que siempre tienen que ver con un determinado sujeto ${ }^{29}$.

La relevancia que adquirió la forma del diálogo gracias a la interpretación de Schleiermacher hizo que surgieran diversas investigaciones en el ámbito de los estudios platónicos en torno a temáticas relacionadas con la 'teoría del diálogo' ${ }^{30}$.

26 Al respecto, véase ibídem p. 131): « Nous pouvons donc à bon droit affirmer que sans compréhension et appropriation de Platon -les deux aspects doivent coexister pour que 'platonisme' il y ait-, l'Idée de l'Herméneutique comme discipline philosophique ne serait pas concevable. De même, le concept de l'herméneutique n'est pas concevable sans platonisme. Il implique de comprendre les discours de l'autre comme pensée qui proclame sa prétention à l'universalité par la communication, et donc comme savoir ».

27 Schleiermacher, Einleitung I, 1 A18: „Ziemlich geringfügig nämlich die Sache behandelnd klagt er, wie ungewiss es immer bleibe bei der schriftlichen Mitteilung der Ideen, ob auch die Seele des Lesers sie selbsttätig nachgebildet und sich also in Wahrheit angeeignet habe, oder ob ihr nur mit dem scheinbaren Verständnis der Worte und Buchstaben eine leere Einbildung gekommen sei, als wisse sie, was sie doch nicht weiss".

28 Ibídem, 1 A18-19: „Seiner Natur nach ist seine Methode eine sokratische gewesen, und zwar, was die ununterbrochen fortschreitende Wechselwirkung und das tiefere Eindringen in die Seele des Hörenden betrifft (...). Daher schon und deswillen die dialogische Form, als notwendig zur Nachahmung jenes ursprünglichen gegenseitigen Mitteilens, auch seinen Schriften eben so unentbehrlich und natürlich ist, als seinem mündlichen Unterrichte".

29 Ibídem, 1, 59: „Als das Äussere dieser Platonischen Form und ihr fast unentbehrliches Schema ist schon oben die dialogische Einkleidung angegeben, nur so aber, wie sie durch lebendige Auffassung jener Absicht den mündlichen Unterricht, der es immer mit einem bestimmten Subjekt zu tun hat, nachzuahmen, noch eine besondere Eigentümlichkeit annimmt, welche erst den Platonischen Dialogen bildet".

30 Para un detallado estado de la cuestión al respecto, véase Erler (Die Philosophie der Antike..., ob. cit., pp. 1-7): „Seine Fokussierung auf die 


\section{La teoría del diálogo a partir de $F$. Schleiermacher}

En especial, la idea de Schleiermacher de que el diálogo constituye el modo esencial de la comunicación filosófica y de que, por consiguiente, goza de una autonomía respecto de otras posibles fuentes de comunicación, ha planteado a la investigación cuestiones que hasta hoy siguen vigentes ${ }^{31}$.

Por ejemplo, si se defiende que los diálogos de Platón exponen una unidad doctrinal, entonces la investigación ha de conciliar los estudios sobre la cronología, autenticidad y estilística de los diálogos para ofrecer una reconstrucción de esa unidad filosófica sistemática ${ }^{32}$. Por otra parte, si se sostiene con Schleiermacher que la forma del diálogo es esencial en la filosofía platónica y al mismo tiempo se intenta una formulación doctrinal de esa filosofía, entonces hay que resolver las contradicciones que presentan sus obras entre sí.

En ocasiones, se ha intentado salvar la disyuntiva del siguiente modo: o bien dando prioridad a la forma del diálogo, lo cual parece bloquear cualquier posible sistematización de la filosofía platónica; o bien dando primacía a la unidad doctrinal y recurriendo a la tesis de que la obra platónica estaría sometida a la evolución del pensamiento de su autor, lo cual parece recluir la forma del diálogo a un mero revestimiento exterior ${ }^{33}$. En contraposición con esta interpretación, estudios procedentes especialmente de la tradición anglo-americana defen-

literarische Form (Dialog) als Teil der inhaltlichen Botschaft und seine Betonung des historischen Zusammen-hanges und des kritischephilologischen Zugriffs (Wiehl 1979; Giuliano 1999) eröffneten einen gerade in jüngster Zeit gerne aufgegriffenen Zugang zu Platon“.

31 Ibídem, p. 1.

32 Por ejemplo, R. Schaerer, La question platonicienne: étude sur les rapports de la pensée et de l'expression dans les Dialogues, Neuchâtel, Secrétariat de l’Université, 1938, que ha tratado el diálogo desde una perspectiva literaria; Clay ("The Origins of the Socratic Dialogue", ob. cit.) y Kahn (Plato and the Socratic Dialogue..., ob. cit.) que han atendido al diálogo como uno de los logoi sokratikoi.

33 En esta línea de interpretación, hay que mencionar a P. Friedländer y a $W$. Jaeger, que polemizan con la interpretación biográfico-genética de Wilamowitz. P. Friedländer (Platón: verdad del ser y realidad de la vida, traducción de S. González Escudero, Madrid, Tecnos, 1989) ha destacado la relevancia del carácter artístico del diálogo, mientras que $\mathrm{W}$. Jaeger, (Paideia: los ideales de la cultura griega, traducción de J. Xirau y W. Roces, México, Fondo de Cultura Económica, 1967) acentúa la unidad orgánica de la obra platónica. dieron la idea de que en los diálogos de Platón se transmite un sistema filosófico y, por consiguiente, es posible su reconstrucción a partir de ellos ${ }^{34}$.

A pesar de estas divergencias, se observa -en líneas generales- la tendencia a considerar en Platón simultáneamente al filósofo y al poeta $^{35}$. En especial, a partir de 1950, ha ganado interés la consideración denominada 'dramática' de la filosofía platónica, en polémica con las interpretaciones 'dogmáticas' ${ }^{36}$, generando en el ámbito de los estudios platónicos investigaciones sobre la forma del diálogo como parte central de la concepción filosófica de Platón ${ }^{37}$.

\section{Bibliografía}

\section{Textos:}

1. Schleiermacher, F.D.E., Über die Philosophie Platos. Geschichte der Philosophie. Vorlesun-

34 Erler (Die Philosophie der Antike..., ob. cit., p. 4): „In angloamerikanischen Bereich bemühten sich seit den 1930er Jahren von G. Russells und G.E. Moores analythischer Philosophie beeinflusste Interpreten (z.B. Hardie 1936; Demos 1939) um eine Analyse der Argumentation in den Dialogen. Einflussreich waren weiterhin die Hinwendung zu sprachlichen Beobachtungen und eine Abkehr von der Metaphysik. Dieser besonders seit den 1930er Jahren in England (Oxford, Cambridge) favorisierte Ansatz brachte zahlreiche wichtige Arbeiten hervor (z.B. Ryle 1939; Robinson 1953; Ross 1953; Cross/Woozley 1964; Lafrance 1971) und machte Schule. Interpreten dieser Richtung belassen der Dialogform oft eine nur dekorative Funktion".

35 Gaiser (Platone come scrittore filosofico..., ob. cit.); J. Dalfen, Polis und Poiesis. Die Auseinandersetzung mit der Dichtung bei Platon und seinen Zeitgenossen, München, Wilhelm Fink Verlag , 1974.

$36 \mathrm{Al}$ respecto, véase Kahn (Plato and the Socratic Dialogue..., ob. cit.) que asume una interpretación 'unitaria' de la obra platónica -la cual se remonta a Schleiermacher-, es decir, entiende que los diálogos (concretamente, los tempranos y medios) no presentan el desarrollo del pensamiento del filósofo, sino la apertura gradual de un plan literario para comunicar su visión filosófica al público en general. Por el contrario, una interpretación 'desarrollista' -introducida por Hermann a inicios del s. XIX-sostiene que, dado que los diálogos son tan diversos en su forma y contenido, Platón habría debido variar su visión filosófica tantas veces como ha cambiado la presentación literaria de su pensamiento. Según Ch. Kahn, la interpretación 'evolucionista' malinterpreta la intención platónica al componer diálogos, ya que esta lectura entiende que en ellos Platón -al igual que otros filósofos escriben tratados y ensayos-expondría la solución a ciertos problemas filosóficos. En cambio, el propósito principal de los diálogos estaría vinculado a una intención ético-pedagógica, a saber, incidir en la orientación moral e intelectual del lector. Por eso, para interpretar los diálogos (tempranos y tardíos) hay que tener en cuenta esta intención de provocar y estimular al lector. Concretamente -afirma el autor- sólo desde el punto de vista de la moral y metafísica definidas en Fedón y República, se puede entender la intención del Platón al componer diálogos como Laques, Cármides, Eutifrón y Protágoras.

37 Griswold (Platonic Writings..., ob. cit.); Nightingale (Genres in Dialogue..., ob. cit.); González (The Third Way..., ob. cit.); Gill/MacGabe (Form and Argument..., ob. cit.). 
gen, über Sokrates und Platon (zwischen 1819 und 1823). Die Einleitungen zur Übersetzung des Platon (1804-1828), hrsg. P. Steiner mit Beiträgen von A. Arndt und J. Jantzen, Hamburg, Felix Meiner Verlag, 1996.

2. Schleiermacher, F.D.E., Hermeneutik und Kritik, Frankfurt am Main, Suhrkamp 1990.

3. Schleiermacher, F.D.E., Los discursos sobre hermenéutica, introducción, traducción y edición bilingüe de L. Flamarique, Cuadernos de Anuario Filosófico, Pamplona, Universidad de Navarra, Servicio de Publicaciones, 1999.

\section{Literatura secundaria:}

4. Arndt, A., "Schleiermacher und Platon“, en F.D.E. Schleiermacher, Über die Philosophie Platos. Geschichte der Philosophie. Vorlesungen, über Sokrates und Platon (zwischen 1819 und 1823). Die Einleitungen zur Übersetzung des Platon (1804-1828), hrsg. P. Steiner mit Beiträgen von A. Arndt und J. Jantzen, Hamburg, Felix Meiner Verlag, 1996.

5. Clay, D., "The Origins of the Socratic Dialogue", en P. Vander Waerdt (ed.), The Socratic movement, Ithaca, Cornell University Press, 1994.

6. Dalfen, J., Polis und Poiesis. Die Auseinandersetzung mit der Dichtung bei Platon und seinen Zeitgenossen, München, Wilhelm Fink Verlag, 1974.

7. Erler, M., Die Philosophie der Antike. Band 2/2. Platon, hrsg. H. Flashar, Überweg, Basel, Schwabe, 2007.

8. Friedländer, P., Platón: verdad del ser y realidad de la vida, traducción de S. González Escudero, Madrid, Tecnos, 1989.

9. Gaiser, K., Platone come scrittore filosofico. Saggi sull'ermeneutica dei dialoghi platonici, Napoli, Istituto italiano per gli studi filosofici, 1984.
10. Gill, C. y McGabe, M. (eds.), Form and Argument in Late Plato, Oxford, Clarendon Press, 1996.

11. González, F. J., The Third Way. New Directions in Platonic Studies, London, Lanham, 1995.

12. Griswold, Ch. (ed.), Platonic Writings. Platonic Readings, New York, Routledge, 1988.

13. Jaeger, W., Paideia: los ideales de la cultura griega, traducción de J. Xirau y W. Roces, México, Fondo de Cultura Económica, 1967.

14. Kahn, Ch., Plato and the Socratic Dialogue. The Philosophical Use of a Literary Form, Cambridge, Cambridge University Press, 1996.

15. Krämer, H.J., Platone e i fondamenti della metafisica. Saggio sulla teoria dei principi e sulle dottrine non scritte di Platone, Milano, Vita e Pensiero, 1993, (4 $4^{\underline{a}}$ ed.).

16. Lamm, J., "Schleiermacher as Plato Scholar", The journal of religion, 80, II, 2000, pp. 206-239.

17. Neschke-Hentschke, A., "Platonisme et tournant herméneutique au début du XIXe siècle en Allemagne", en A. Laks y A. Neschke. (eds.), La naissance du paradigme herméneutique. Schleiermacher, Humboldt, Boeckh, Droysen, Paris, Presses Universitaires de Lille, 1990.

18. Nightigale, A. W., Genres in Dialogue. Plato and the Construct of Philosophy, Cambridge, Cambridge University Press, 1995.

19. Reale, G., Per una nuova interpretazione di Platone. Rilettura della metafisica der grandi dialoghi alla luce delle 'Dottrine non scritte', Milano, Vita e Pensiero, 1991, (11- ed.).

20. Rowe, Ch., Plato, Brighton, Harvester, 2003.

21. Schaerer, R., La question platonicienne: étude sur les rapports de la pensée et de l'expression dans les Dialogues, Neuchâtel, Secrétariat de l’Université, 1938.

22. Thesleff, H., Studies in Platonic chronology, Helsinki, Societas Scientarum Fennica, 1982. 
